\title{
Technologies for recovery and reuse of plant nutrients from human excreta and domestic wastewater: a protocol for a systematic map and living evidence platform
}

Biljana Macura ${ }^{*}$ (D), James Thomas ${ }^{2}$, Geneviève S. Metson³ ${ }^{3}$ Jennifer R. McConville ${ }^{4}$, Solveig L. Johannesdottir ${ }^{5}$, Dan Seddon ${ }^{6}$ and Robin Harder ${ }^{4}$

\begin{abstract}
Background: Research and development on the recovery and reuse of nutrients found in human excreta and domestic wastewater has intensified over the past years, continuously producing new knowledge and technologies. However, research impact and knowledge transfer are limited. In particular, uptake and upscaling of new and innovative solutions in practice remain a key challenge. Achieving a more circular use of nutrients thus goes beyond technological innovation and will benefit from a synthesis of existing research being readily available to various stakeholders in the field. The aim of the systematic map and online evidence platform described in this protocol is threefold. First, to collate and summarise scientific research on technologies that facilitate the recovery and reuse of plant nutrients and organic matter found in human excreta and domestic and municipal wastewater. Second, to present this evidence in a way that can be easily navigated by stakeholders. Third, to report on new relevant research evidence to stakeholders as it becomes available.
\end{abstract}

Methods: Firstly, we will produce a baseline systematic map, which will consist of an extension of two previous related syntheses. In a next stage, with help of machine learning and other automation technologies, the baseline systematic map will be transformed into 'living mode' that allows for a continually updated evidence platform. The baseline systematic map searches will be performed in 4 bibliographic sources and Google Scholar. All searches will be performed in English. Coding and meta-data extraction will include bibliographic information, locations as well as the recovery and reuse pathways. The living mode will mostly rely on automation technologies in EPPI-Reviewer and the Microsoft Academic database. The new records will be automatically identified and ranked in terms of eligibility. Records above a certain 'cut-off' threshold will be manually screened for eligibility. The threshold will be devised based on the empirically informed machine learning model. The evidence from the baseline systematic map and living mode will be embedded in an online evidence platform that in an interactive manner allows stakeholders to visualise and explore the systematic map findings, including knowledge gaps and clusters.

Keywords: Circular economy, Nitrogen, Nutrient recovery, Phosphorus, Resource recovery, Sewage

*Correspondence: biljana.macura@sei.org

${ }^{1}$ Stockholm Environment Institute, P.O. Box 24218, 10451 Stockholm, Sweden

Full list of author information is available at the end of the article

\section{Background}

In recent years, the concepts of 'nutrient circularity', 'closing the nutrient loop', 'circular nutrient solutions', and 'circular nutrient economy' have gained traction [11, 19, 21, 24]. This echoes the increasing understanding that, original author(s) and the source, provide a link to the Creative Commons licence, and indicate if changes were made. The images or other third party material in this article are included in the article's Creative Commons licence, unless indicated otherwise in a credit line to the material. If material is not included in the article's Creative Commons licence and your intended use is not permitted by statutory regulation or exceeds the permitted use, you will need to obtain permission directly from the copyright holder. To view a copy of this licence, visit http://creativecommons.org/licenses/by/4.0/. The Creative Commons Public Domain Dedication waiver (http://creativeco mmons.org/publicdomain/zero/1.0/) applies to the data made available in this article, unless otherwise stated in a credit line to the data. 
in order to mitigate nutrient pollution in water bodies and improve global nutrient security, societies around the world have to learn how to better recover nutrients from organic residuals for reuse in agriculture [16]. These organic residuals can include crop and food remains and animal and human manure.

Research and development on nutrient recovery and reuse has intensified over the past years [8, 10, 22], but research impact and knowledge transfer to policy and practice are limited. In particular, upscaling of new and innovative solutions in practice remain a key challenge $[1,2]$. This is because issues of environmental and resource management are often related to governance [7] and subject to cultural, economic, institutional, and regulatory barriers $[3,17]$. Achieving a more circular use of nutrients thus goes beyond technological innovation and requires engagement with civil society and better translation of scientific findings into policy and practice.

One of the key obstacles for taking a full advantage of the nutrient and carbon recovery and reuse potential is that existing knowledge is scattered across different sources and is rapidly growing. Therefore, it is difficult for actors even within the same sector or country to navigate existing knowledge, let alone to keep track of new findings. Moreover, as data from different sources is likely to be reported in different formats and according to different conceptual models, significant effort is required before the knowledge can be used to inform decisions. A trusted open-access database that compiles and consolidates best available scientific evidence in a systematic and easily accessible manner is needed to support decisions related to safely and adequately recirculating nutrients and carbon in cities and rural areas.

\section{Previous evidence syntheses}

There are numerous reviews that summarise a subset of the literature on recycling nutrients from human excreta and domestic wastewater to agriculture. Typically, these reviews have focused on a specific: nutrient, e.g., phosphorus [6]; recovery technology, e.g., struvite precipitation [20]; input stream, e.g., digestate [25]; or combination thereof, e.g., struvite precipitation from digestate $[13,14]$.

Other reviews were more comprehensive in scope. For instance, Harder et al. reviewed different options for recycling nutrients contained in human excreta and domestic wastewater to agriculture in terms of pathways, processes, and products more broadly (hereafter referred to as 'SANAGRI' review). Two recent systematic maps collated research on ecotechnologies for recovery and reuse of carbon and nutrients from domestic wastewater [10] and agricultural waste streams [15], but were limited to literature published between 2013 and 2017 (hereafter referred to as 'BONUS RETURN' reviews). The overlap in included studies between the SANAGRI and BONUS RETURN reviews was surprisingly low and just under ten percent. Moreover, there seems to be a substantial number of relevant studies yet to be collated.

\section{Objectives of the review}

The aim of the systematic map and living evidence platform is to: (1) update and extend the SANAGRI and BONUS RETURN reviews on technologies that facilitate the recovery of plant nutrients from human excreta and domestic wastewater for reuse in agriculture; (2) set up an online evidence platform that enables stakeholders to navigate existing evidence and that reports on new relevant research as it becomes available; (3) explore procedures to effectively and continuously update the evidence base in the future and optimise search strings for this subject.

The primary question for this systematic map and evidence platform is:

What evidence exists on technologies for the recovery of plant nutrients from human excreta and domestic wastewater for reuse in agriculture?

This question can be broken down into following elements:

- Population(s): systems that manage human excreta (i.e., urine and faeces), streams containing human excreta (e.g., yellowwater, brownwater, blackwater, domestic and municipal wastewater), or residues and products that are derived from these streams (e.g., digestate, faecal or sewage sludge, treated effluent).

- Intervention(s): practices and technologies undertaken for the purpose of recovering plant nutrients, including organic matter.

- Outcome(s): recovered product containing plant nutrients (with or without organic matter) suitable for reuse in agriculture, or to produce fertilizers.

The key outputs of this work will be as follows:

1. A detailed searchable database of relevant studies, including:

a. A description of recovery pathways (sensu Harder et al. [8]. A recovery pathway describes what is being reused or recovered, from what, and how. A pathway is characterized by the following descriptors: at least one source stream (e.g., municipal wastewater) and access stream (e.g., sewage sludge ash), a sequence of processes (e.g., leaching followed by precipitation), and at least one recovery product (e.g., struvite). 
b. Other relevant metadata such as bibliographic information, study type, scale, and location.

2. An evidence platform comprised of:

a. A user-friendly interface to search for relevant evidence, organised by topic, pathway, study type, recycled product, etc.

b. An 'evidence atlas', that is, an interactive geographical map visualising the location of author affiliations (and study locations, if resources allow).

c. A series of 'heat maps' that cross-tabulate two descriptors (e.g., process versus product, or process versus source stream). These heat maps will be used to systematically identify knowledge clusters (i.e., subtopics that are well-represented by research studies) and knowledge gaps (i.e., subtopics that are un- or under-represented by research studies).

3. A suite of living mode procedures to keep the database up to date, including machine learning support to search and screen new research as it becomes available.

\section{Methods}

The systematic mapping process will follow the Collaboration for Environmental Evidence guidelines and standards for evidence synthesis in environmental management [5] and it conforms to ROSES reporting standards (see Additional file 1). Guidance for the production and publication of Cochrane living systematic reviews [4] was also used to inform the process.

The mapping process will be comprised of several stages. In the first stage, we will produce a baseline systematic map, which will consist of an extension of our previous work $[8,10,15]$. In a second stage, with help of machine learning and other automation technologies for searching, screening and coding, the baseline review will be transformed into living mode to allow for a continually updated systematic map that incorporates relevant new evidence as it becomes available. Finally, the evidence from the baseline systematic map and living mode will be embedded in an online evidence platform that allows stakeholder to explore the systematic map findings in an interactive manner. Any changes to the protocol will be described and justified in the final report.

The mapping process with living mode and the development of the evidence platform are illustrated in Fig. 1 and outlined in the rest of the protocol.

\section{Codesign process and stakeholder engagement}

A codesign process with continuous stakeholder input will be used throughout the development of the systematic map and evidence platform to assure relevance of the review findings for stakeholders, legitimacy of the review process, and better evidence uptake into policy and practice [12]. Involved stakeholders are being identified using the team's knowledge and contacts, snowballing, systematic searching and open online comment periods. Although we will be initially engaging mostly with Swedish researchers and practitioners, our ultimate aim is to work with stakeholders internationally and with practitioners in Sweden.

We have designed multiple points of entry for stakeholder engagement (see Fig. 2).

\section{Review scope}

In order to understand interest for this map and evidence platform, the research team administered a survey during June and July 2020 to the Swedish Nutrient Platform (SNP) network and Swedish experts known to the team. Survey questions were initially piloted at an SNP workshop and narrowed in scope before circulation of a wider survey within our stakeholder network. Respondents were asked to evaluate the relevance and usefulness of three potential functions of an evidence platform:

- A description of technological solutions or practices for nutrient reuse and recovery with details about relevant pathways.

- Technical details and performance of these technological solutions.

- Information about relevant actors, including networks, researchers and developers in this field.

Over $55 \%$ of respondents thought all three functions could be useful for their work or to their organizations in general. Moreover, when asked what types of stakeholders might benefit from such an evidence platform, the wastewater utilities, government agencies, and researchers were the top three groups of actors identified as the platform beneficiaries (although many more were mentioned). This information has been used in the design of this initial protocol.

\section{Protocol design}

Additional input on the scope of the mapping and on future engagement strategies for design of the platform was solicited through a review of this initial protocol. During a 3-week open consultation process we reachedout to a wide network of actors for comments. The protocol was shared on the project website (http://www.endof 


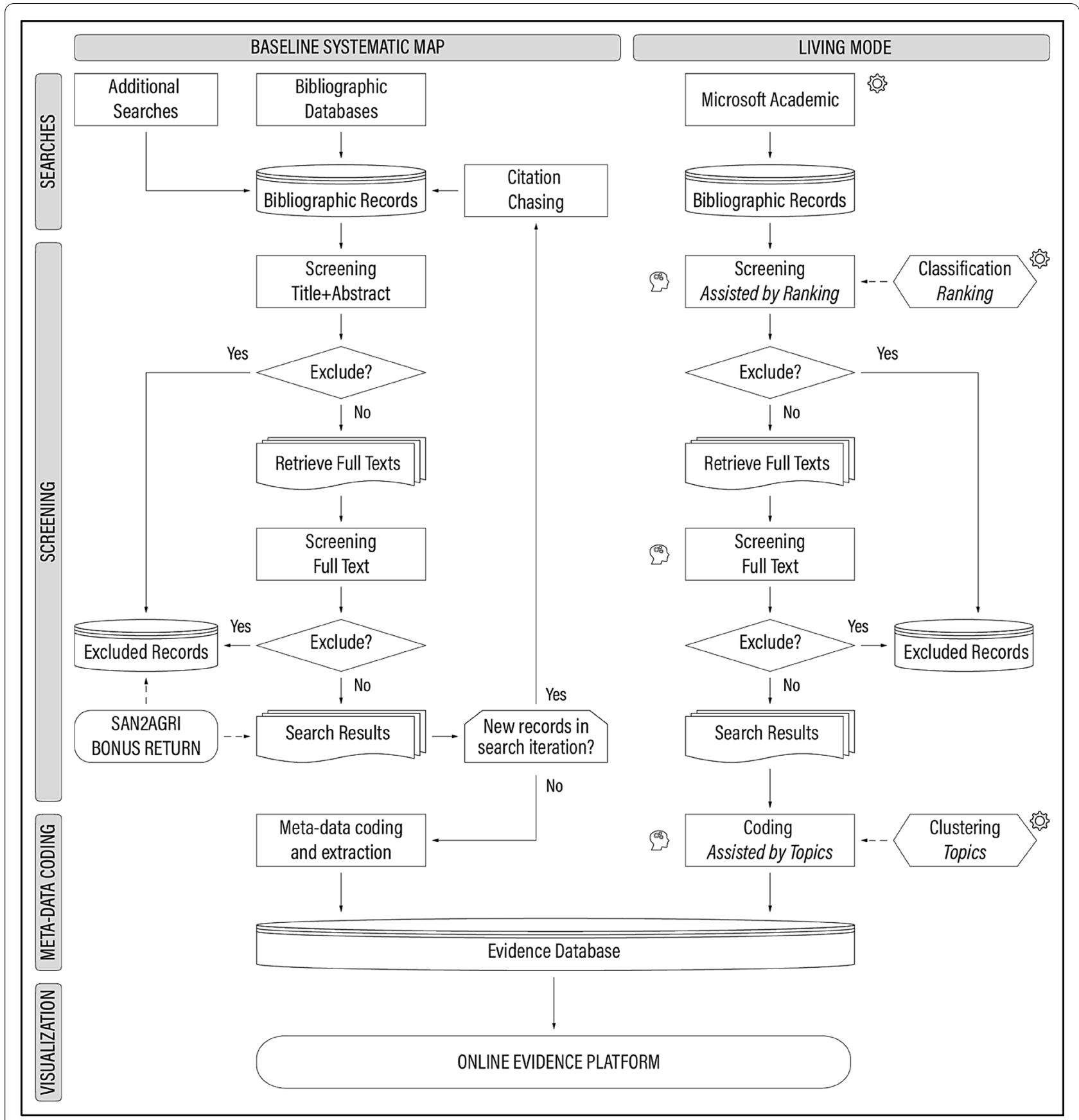

Fig. 1 Flow diagram for the development of the systematic map and evidence platform

wastewater.net/), via the Swedish Nutrient Platform (SNP, https://www.ri.se/sv/svenskanaringsplattformen) and other similar networks that connect subject experts, decision makers and others interested actors. Stakeholders were invited to comment on the scope, search strategy, meta-data coding structure or provide any other feedback. The feedback were collect via a survey form which has specific questions related to the above points but also allows for free text answers. The protocol was updated following this process. Additional file 2 contains summary of survey responses.

\section{Evidence platform design}

We will invite representatives of academia, farmers, utilities and government agencies to test and help design the interface of the evidence platform. Stakeholder input and 


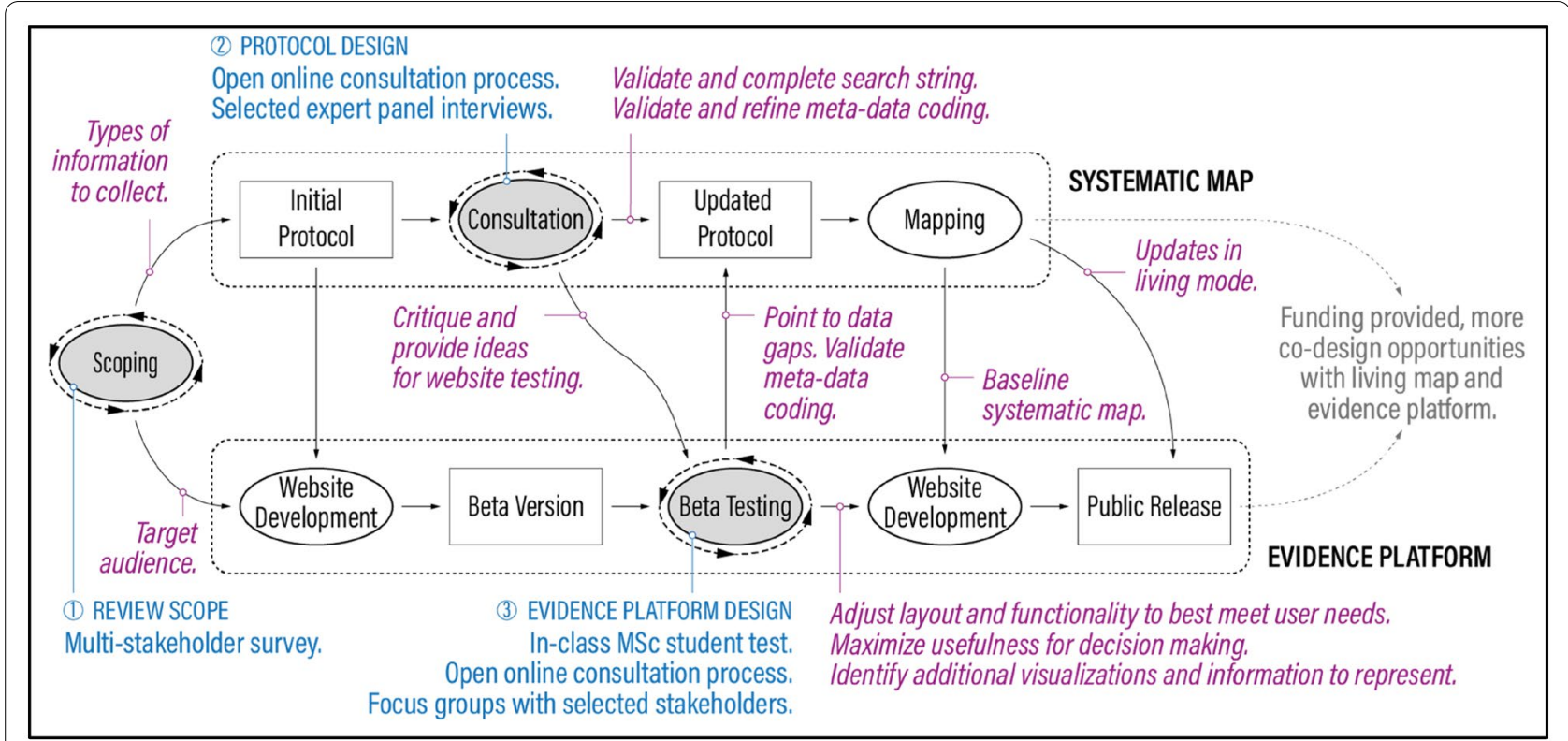

Fig. 2 Overview of the codesign process supporting the development of the protocol, systematic map, and evidence platform. The upper part of the figure describes the codesign of the systematic mapping process, the lower part of the evidence platform. Ovals represent processes and squares are resulting products. Text in blue denotes types of interaction with stakeholders, and text in magenta describes types of expected input from stakeholders

feedback will be used to ensure usefulness of the platform to potential users. We will combine three methods for improving the platform's design: focus groups, betatesting and open feedback process. Focus groups (with 3 to 4 participants) will be organised in autumn 2021. The participants will be survey respondents (12 expressed interest) and practitioners and experts in waste management and food systems identified through the previous codesign stages. Focus groups will be asked to use the platform and give feedback on comprehensiveness, ease of use, data organization and visualization options, as well as if/how the information can help in decision making. The focus group setting allows for different types of feedback as participants can build on each other's experiences. Using the platform during a focus group will also allow the research group to observe how people navigate the platform and see if there are sticking points. Because academics were highlighted as an important potential user, we will also organise a beta testing session with graduate students (MSc level) as part of a class. The beta version of the platform will also be available online for open testing and commenting during a 4-week period. Users will be able leave the comments via a feedback form. Finally, we plan to have continued open dialogue with interested users after the platform is launched to continue improving it. There will be a comments and questions section on the website but pending funding and interest we also hope to continue more dynamic engagement to update the evidence platform as well as the user interface over time to further match user needs.

\section{Search strategy \\ Searches for baseline systematic map}

Here we describe search strategy for the baseline systematic map. The review will merge the datasets of the SANAGRI [8] and BONUS RETURN [10, 15] reviews and continue adding new search records from several sources as described below. Searches will not be restricted to any time period.

Bibliographic searches We will search for evidence in the following bibliographic databases and search platforms:

1. Scopus

2. Web of Science Core Collections (consisting of the following indexes: SCI-EXPANDED, SSCI, A\&HCI, CPCI-S, CPCI-SSH, and ESCI)

3. The ProQuest Dissertation and Theses

4. Microsoft Academic

Searches will be performed using English language search terms. Subscriptions from the Swedish University of Agricultural Sciences and Stockholm University will be used to access subscription services above (such as Web of Science). 
Search strings The search string will be composed of four substrings described in Table 1 . The string builds on the experience from previous reviews $[8,10,15]$ and it includes a combination of population, intervention and outcome terms. In addition, to avoid literature from pharmacology, medicine and veterinary science, we have added a set of exclusion terms (D). Taken together, the final string is combined as follows: A AND B AND C AND (NOT D). Depending on search functionalities, the search string is adapted to each bibliographic source and details are available in Additional file 3.

During search process, we will filter out medical and veterinary journals. During the screening process, we will filter out on related terms such as: veterinary, metabolomic, kidney, pharmacology and similar. The list of excluded journals and terms will be updated during the review process and published in the final report.

Citation chasing Records that are cited by eligible records indexed in Scopus and Web of Science will be retrieved and added to the search results (i.e., backward citation chasing). Moreover, the reference lists of all relevant reviews found during the systematic mapping process will be searched for eligible studies. If possible, we will also retrieve records that cite eligible records (i.e., forward citation chasing) as this information is obtainable from Scopus (using 'cited by' functionality) and Web of Science (via Cited Reference Search option). Citation chasing will be implemented as an iterative process. After each iteration duplicates will be removed, and the process will stop once no new records are found.

Additional searches The BONUS RETURN reviews [10, 15] included extensive searches for grey literature, but the contribution of grey literature in English to the evidence base was minor (for example, out of 448 articles included in the evidence base of a systematic map on recycling of carbon and nutrients from domestic wastewater, only 3 relevant reports in English were found and included). This review will conduct only searches on Google Scholar, but no specialist websites will be searched (see Additional file 3 for details). Nevertheless, an effort will be made to map case studies that include real application of reuse and recovery technologies in Sweden. Moreover, we will contact other experts and our stakeholder group for relevant research (see "Data coding strategy for baseline systematic map" Section) and the calls for evidence will be issued on Twitter, ResearchGate, and similar platforms.

Testing comprehensiveness of searches During the scoping phase, search results were screened against a benchmark list including articles of known relevance to the review to examine whether these searches are able to locate relevant evidence (see Additional file 3). In cases where relevant articles from the benchmark list were not found with the search strategy, the search strings were examined to identify why articles were missed. Search strings were then adapted where relevant. The final search string includes all articles from the benchmark list.

Assembling a library of search results Results of the bibliographic searching will be combined, and duplicates will be removed prior to screening. A library of search results will be assembled in EPPI-Reviewer [23].

\section{Searches in living mode}

We will keep our evidence base up to date with the help of the Microsoft Academic database (https://academic. microsoft.com). To test comprehensiveness of this database, we have checked if it contains records from SANAGRI [8] and the BONUS RETURN wastewater [10] reviews. Out of 2904 records in total from these studies, only 94 (3.2\%) were not indexed on Microsoft Academic (neither on Scopus nor Web of Science-the two main literature sources for the baseline map), which is why we will consider using this as the only search source for living mode. We will re-assess the comprehensiveness of Microsoft Academic however upon completion of the baseline systematic map.

Two approaches will be combined to conduct searches on Microsoft Academic. The first approach consists of conducting standard Boolean searches on Microsoft Academic done within EPPI-Reviewer. The second approach uses a newly developed machine learning feature in

Table 1 Search substrings (shown as formatted for Web of Science) 
EPPI-Reviewer. EPPI-Reviewer receives a new copy of the Microsoft Academic dataset every two weeks. The team at the EPPI-Centre, in conjunction with a team from Microsoft, has developed machine learning tools that can 'learn' the scope of a given review, and automatically identify newly published and potentially relevant studies each time a new version of the Microsoft Academic dataset becomes available. Even though there is likely to be substantial overlap between the machine learning and Boolean search results, the set of records that will result from the combination of the two approaches will be free of duplicates and will be combined into a unified list. We will be testing sensitivity by comparing numbers of missed records between the two approaches.

\section{Article screening and study eligibility criteria Screening for baseline systematic map}

Screening will be done at two levels: at title and abstract (screened concurrently for efficiency) and at full text. Potentially relevant abstracts will be retrieved, tracking those that cannot be located or accessed and reporting these in the final review. Retrieved records will then be screened at full text, with each record being assessed by one experienced reviewer.

Prior to commencing screening, consistency checking will be performed with all reviewers on a subset of articles at both title and abstract and full text levels. A subset of approximately $10 \%$ of title and abstract records and full text records will be independently screened by all reviewers. The results of the consistency checking will then be compared among reviewers and all disagreements will be discussed in detail. Where the level of agreement among reviewers is low (below 80\%), further consistency checking will be performed on an additional set of articles. This will be repeated until the consistency level reaches at least $80 \%$.

We will provide a list of articles excluded at title and abstract, and at full text, with reasons for exclusion in the final report. Reviewers who have also authored articles to be considered within the review will be excluded from decisions regarding inclusion of their own work.

\section{Screening in living mode}

For the living mode, we will explore how eligible records could be identified automatically using machine learning algorithms available in EPPI-reviewer. A training set will be compiled from records that were manually screened based on titles and abstracts by at least two reviewers. Based on the training set, the system will build bespoke classifiers which will rank records in order of their expected eligibility. This means that it will be possible to tailor a machine learning threshold below which a record is unlikely to be relevant. Records below this 'cut-off' threshold could be automatically excluded while records above the threshold would have to undergo manual screening. In case the number of new potentially relevant records exceeds the capacity for manual review, reviewing only those ranked highest will help maximise the yield of relevant records identified within available resource.

At the moment, EPPI-reviewer only has machine learning technology for classifying and screening records based on information available in title and abstracts, but not based on full texts. Therefore, full text screening (and consequently also coding) will still need to be performed manually in the living mode.

\section{Eligibility criteria}

The following criteria will be applied at all levels of screening and for both baseline review and living mode:

- Eligible population(s): systems that manage human excreta or streams containing human excreta, notably domestic and municipal wastewater. This includes systems that manage residues and products that are derived from human excreta or wastewater that contains human excreta, such as digestate, sewage sludge, treated effluent, etc. Synthetic wastewater intended to simulate aforementioned streams will be included. Both municipal and on-site systems are relevant, as well as co-treatment with other organic residuals. Systems that manage only greywater, stormwater, industrial wastewater (e.g., tannery wastewater), agricultural wastewater (e.g., milling wastewater) or animal manure will not be considered.

- Eligible intervention(s): any technology or practice undertaken for the purpose of facilitating the recirculation of plant nutrients, and possibly organic matter, to agriculture. Recirculation can take place either through direct reuse after treatment of human excreta or streams containing human excreta, or through products derived from the extraction of nutrients from human excreta or streams that contain or derive from human excreta. Practices that are undertaken for the purpose of recovering carbon (for instance as methane for energy purposes or as polyhydroxyalkanoate for producing bioplastics) and water (for instance for irrigation or industrial purposes) are excluded unless the practice allows for simultaneous nutrient recovery or reuse.

- Eligible outcome(s): product that contains plant nutrients, with or without organic matter, and is suitable for reuse in agriculture, or as raw material to produce fertilizers. 
- Eligible study type(s): primary research and reviews that describe nutrient recovery technologies or the reuse of recovered nutrients in agriculture. In addition to experimental studies at the lab, bench, pilot or full scale, this also includes human health risk and sustainability assessments, as well as studies on user acceptance.

- Eligible languages: English.

- Time frame: no time limitations will be applied.

\section{Study validity assessment}

The validity of studies will not be appraised in detail as part of this systematic map, which is in accordance with accepted systematic mapping methodological guidance [9]. Nevertheless, we will map indicators of study validity such as elements of study design including study scale and type (see Data coding strategy) that will be further developed during the review process.

\section{Data coding strategy}

\section{Data coding strategy for baseline systematic map}

The meta-data to be extracted for all eligible studies include bibliographic information, study context and design as well as the recovery pathways. A recovery pathway describes the combination of a primary input, treatment train, and a product (see Fig. 3 for additional definitions). Details of meta-data to be coded are specified in Table 2; Additional file 4.
Meta-data extraction and coding will be performed by multiple reviewers following consistency checking on a subset of up to 30 full texts, discussing all disagreements and clarifying coding scheme where needed.

If resources allow, we may contact authors by email with requests for missing information or clarifications. Whenever information was to be retrieved in other ways than directly from the document, this will be annotated and reported in the final review.

\section{Data coding strategy for living mode}

It is currently not possible to consistently and comprehensively code full texts using automation technologies. Therefore any automated meta-data coding will have to rely on information present in the title, abstract, and keywords. We will also be testing clustering of records using the Microsoft Academic topics (including parent, child and related topics representing topic hierarchy and connections) that are available for each record in the database. These topics are automatically generated using topic modelling on the basis of full texts. Manual data coding will be done as shown in the "Data coding strategy for baseline systematic map" Section.

\section{Study mapping and presentation Systematic map report}

The evidence base identified within the map will be described primarily within a systematic map database; a searchable (relational) database with columns containing codes and meta-data related to the variables described in

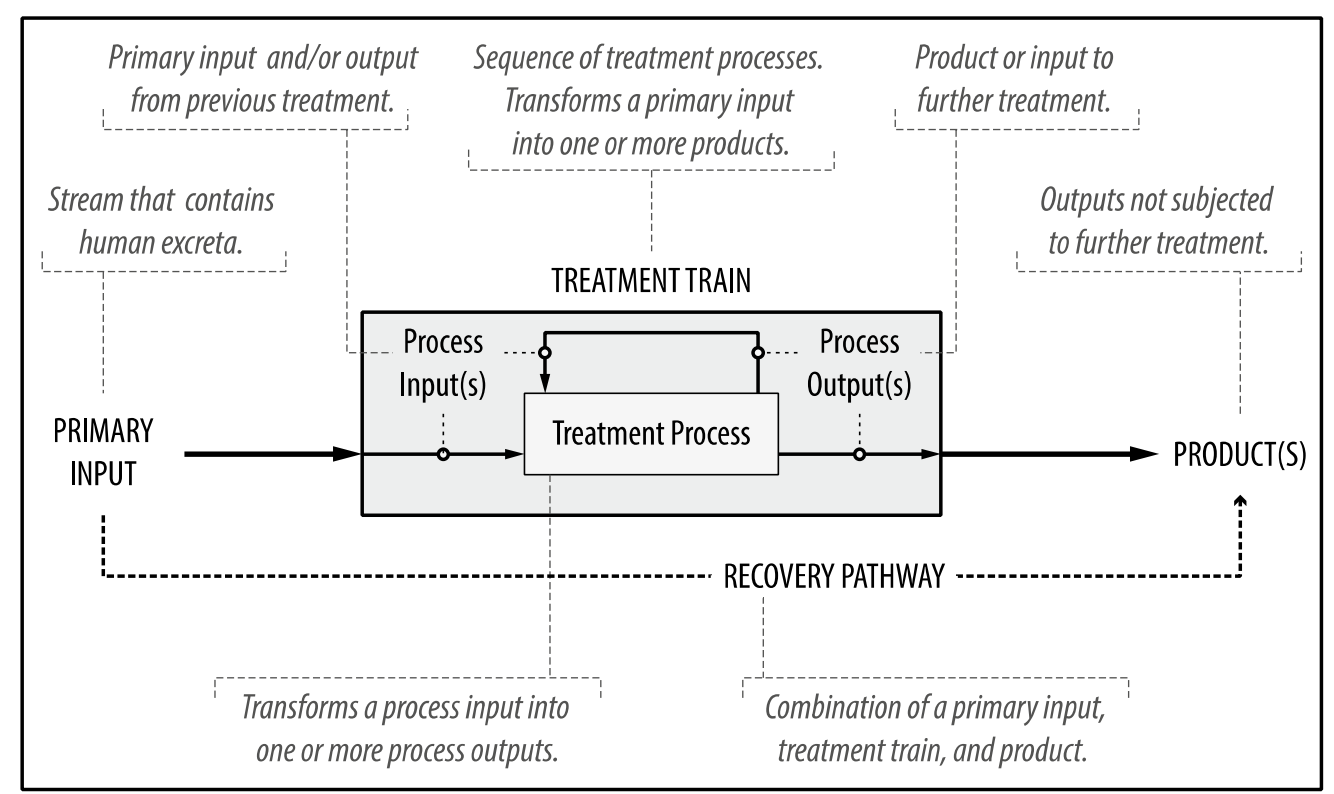

Fig. 3 Definition of key terms used for meta-data coding 
Table 2 Overview of meta-data to be coded

\begin{tabular}{lll}
\hline Meta-data domains & Possible values & Comment \\
\hline $\begin{array}{ll}\text { Primary input(s) } \\
\text { Process(es) }\end{array}$ & $\begin{array}{l}\text { e.g., urine, blackwater, sewage sludge ash, treated effluent } \\
\text { e.g., selective crystallization, hydrothermal liquefaction, sorption, membrane } \\
\text { filtration, etc. }\end{array}$ & Where applicable \\
Product(s) & e.g., struvite, ammonium sulfate, algal biomass, etc. & Where applicable \\
Commercial name of process & e.g., NuReSys, DHV Crystallactor, etc. & Where applicable \\
Commercial name of product & e.g., Aurin, Crystal Green, etc. & For experimental studies only \\
Author affiliation country and location & Country and city, including longitude and latitude of the city & \\
Study location & Country and city, including longitude and latitude of the city & \\
Study scale & Lab, bench, pilot, or full scale & \\
Study type & Recovery process, recovery product, reuse in agriculture, sustainability assess- & \\
Research type & ment, user acceptance & \\
Publication source & Primary (experimental or modelling studies) or secondary (literature reviews) & \\
\hline
\end{tabular}

the meta-data extraction and coding schema, above. In addition, we will produce heat maps that cross tabulate two variables and detail the volume of evidence (number of studies) within each cell of the table. Various combinations of variables will be used for these heat maps, including pathway components and similar meta-data as per Table 2 . The heatmaps will be used to identify knowledge clusters (well-represented subtopics that are amenable to full synthesis via systematic review) and gaps (un- or underrepresented topics). Identification will be performed by visual inspection by a methodology expert of the review team (i.e., not a subject expert to avoid preconception bias). The gaps and clusters will then be validated with stakeholders in focus group discussions.

\section{Visualising the systematic map findings via an evidence platform}

Evidence syntheses and knowledge brokering tools work at the boundary between science and policy and thus have to meet user needs [18]. To increase the use and uptake of evidence, the findings of this systematic map will be visualized through an evidence platform continuously developed through a codesign process with stakeholders (see "Codesign process and stakeholder engagement" Section).

\section{Maintaining the living evidence platform}

After the baseline review is finalised, and once the living mode is set up, the database will be automatically populated and uploaded to the evidence platform every 6 months or when there are more than 50 new records found and up until the year 2026. The process will then be revised to account for technology improvements. After every update, a short report will be produced describing records added to the database.

\section{Supplementary Information}

The online version contains supplementary material available at https://doi. org/10.1186/s13750-021-00235-x.

Additional file 1: ROSES form for systematic map protocols.

Additional file 2: Stakeholder survey with summary of responses.

Additional file 3: Scoping exercise, benchmark list and search strategy.

Additional file 4: Coding scheme.

\section{Acknowledgements}

We are grateful to all stakeholders for provided feedback and suggestions and our advisory board members, Neal Haddaway and Erik Kärrman, for their valuable inputs.

\section{Authors' contributions}

$\mathrm{BM}$ and $\mathrm{RH}$ drafted initial version of the manuscript, edited and finalised the manuscript. JT wrote and contributed to sections about living mode procedures, GM and JM wrote and contributed to sections about stakeholder engagement and co-design. DS contributed to the design of evidence platform. SJ read and edited the manuscript. All authors read and approved the final manuscript.

\section{Funding}

This protocol was developed as part of the project "End-of-wastewater", which has received funding from the Kamprad Family Foundation under grant agreement 20200021. Early stakeholder engagement took place as part of the project "Going circular", which has received funding from the Swedish Research Council for Environment, Agricultural Sciences and Spatial Planning (Formas) under grant agreement 2019-02476.

\section{Availability of data and materials}

Not applicable.

\section{Declarations}

Ethics approval and consent to participate Not applicable.

Consent for publication

Not applicable.

\section{Competing interests}

The authors declare they have no competing interests. 


\section{Author details}

1'Stockholm Environment Institute, P.O. Box 24218, 10451 Stockholm, Sweden. ${ }^{2}$ Department of Social Science, EPPI-Centre, UCL Institute of Education, 21, London, UK. ${ }^{3}$ Department of Physics, Chemistry and Biology, Linköping University, 58183 Linköping, Sweden. ${ }^{4}$ Department of Energy and Technology, Swedish University of Agricultural Sciences, P.O. Box 7032, 75007 Uppsala, Sweden. ${ }^{5}$ RISE Research Institutes of Sweden, Ultunaallén 4, 75651 Uppsala, Sweden. ${ }^{6}$ Nature-Based Solutions Initiative, Department of Zoology, University of Oxford, Oxford, UK.

Received: 20 May 2021 Accepted: 19 August 2021

Published online: 28 August 2021

\section{References}

1. Andersson K, Dickin S, Rosemarin A. Towards "sustainable" sanitation: challenges and opportunities in urban areas. Sustain. 2016. https://doi.org/10. 3390/su8121289.

2. Andersson $\mathrm{K}$, Otoo M, Nolasco M. Innovative sanitation approaches could address multiple development challenges. Water Sci Technol. 2018;77:855-8. https://doi.org/10.2166/wst.2017.600

3. Barquet $K$, Järnberg L, Rosemarin A, Macura B. Identifying barriers and opportunities for a circular phosphorus economy in the Baltic Sea region. Water Res. 2020. https://doi.org/10.1016/j.watres.2019.115433.

4. Brooker J, Synnot A, McDonald S, Elliott J, Turner T et al. Guidance for the production and publication of Cochrane living systematic reviews: Cochrane Reviews in living mode Version December 2019. Cochrane. Available from: https:/community.cochrane.org/sites/default/files/uploa ds/inline-files/Transform/201912_LSR_Revised_Guidance.pdf.

5. Collaboration for Environmental Evidence. 2018. Guidelines and Standards for Evidence synthesis in Environmental Management. Version 5.0. In: Pullin AS, Frampton GK, Livoreil B, Petrokofsky G, Editors. www.envir onmentalevidence.org/information-for-authors. Accessed 1 Mar 2021.

6. Egle L, Rechberger $\mathrm{H}$, Zessner M. Overview and description of technologies for recovering phosphorus from municipal wastewater. Resour Conserv Recycl. 2015;105:325-46. https://doi.org/10.1016/j.resconrec. 2015.09.016.

7. Hackmann H, Moser SC, St. Clair AL. The social heart of global environmental change. Nat Clim Chang. 2014;4:653-5. https://doi.org/10.1038/ nclimate2320

8. Harder R, Wielemaker R, Larsen TA, Zeeman G, Öberg G. Recycling nutrients contained in human excreta to agriculture: pathways, processes, and products. Crit Rev Environ Sci Technol. 2019. https://doi.org/10.1080/ 10643389.2018.1558889.

9. James KL, Randall NP, Haddaway NR. A methodology for systematic mapping in environmental sciences. Environ Evid. 2016;5:1-13. https://doi. org/10.1186/s13750-016-0059-6.

10. Johannesdottir SL, MacUra B, McConville J, Lorick D, Haddaway NR, Karczmarczyk A, Ek F, Piniewski M, Księżniak M, Osuch P. What evidence exists on ecotechnologies for recycling carbon and nutrients from domestic wastewater? A systematic map. Environ Evid. 2020;9:1-14. https://doi.org/ 10.1186/s13750-020-00207-7.

11. Koppelmäki K, Helenius J, Schulte RPO. Nested circularity in food systems: a Nordic case study on connecting biomass, nutrient and energy flows from field scale to continent. Resour Conserv Recycl. 2021. https://doi. org/10.1016/j.resconrec.2020.105218.

12. Land M, Macura B, Bernes C, Johansson S. A five-step approach for stakeholder engagement in prioritisation and planning of environmental evidence syntheses. Environ Evid. 2017. https://doi.org/10.1186/ s13750-017-0104-0.
13. Lorick D, Macura B, Ahlström M Grimvall A, Harder R. Effectiveness of struvite precipitation and ammonia stripping for recovery of phosphorus and nitrogen from anaerobic digestate: a systematic review. Environ Evid. 2020;9:1-20. https://doi.org/10.1186/s13750-020-00211-x.

14. Macura B, Johannesdottir SL, Piniewski M, Haddaway NR, Kvarnström E. Effectiveness of ecotechnologies for recovery of nitrogen and phosphorus from anaerobic digestate and effectiveness of the recovery products as fertilisers: a systematic review protocol. Environ Evid. 2019;8:1-9. https://doi.org/10.1186/s13750-019-0173-3.

15. Macura B, Piniewski M, Księżniak M, Osuch P, Haddaway NR, Ek F, Andersson K, Tattari S. Effectiveness of ecotechnologies in agriculture for the recovery and reuse of carbon and nutrients in the Baltic and boreotemperate regions: a systematic map. Environ Evid. 2019;8:1-18. https:// doi.org/10.1186/s13750-019-0183-1.

16. McConville J, Drangert JO, Tidåker P, Neset TS, Rauch S, Strid I, Tonderski K. Closing the food loops: guidelines and criteria for improving nutrient management. Sustain Sci Pract Policy. 2015;11:33-43. https://doi.org/10. 1080/15487733.2015.11908144.

17. McConville JR, Kvarnström E, Jönsson H, Kärrman E, Johansson M. Source separation: challenges and opportunities for transition in the Swedish wastewater sector. Resour Conserv Recycl. 2017;120:144-56. https://doi. org/10.1016/j.resconrec.2016.12.004.

18. McNie EC. Reconciling the supply of scientific information with user demands: an analysis of the problem and review of the literature. Environ Sci Policy. 2007. https://doi.org/10.1016/j.envsci.2006.10.004.

19. Nesme T, Withers PJA. Sustainable strategies towards a phosphorus circular economy. Nutr Cycl Agroecosyst. 2016;104:259-64. https://doi.org/10. 1007/s10705-016-9774-1.

20. Rahman MM, Salleh MAM, Rashid U, Ahsan A, Hossain MM, Ra CS. Production of slow release crystal fertilizer from wastewaters through struvite crystallization—a review. Arab J Chem. 2014;7:139-55. https://doi.org/10. 1016/j.arabjc.2013.10.007

21. Robles Á, Aguado D, Barat R, Borrás L, Bouzas A, Giménez JB, Martí N, Ribes J, Ruano MV, Serralta J, Ferrer J, Seco A. New frontiers from removal to recycling of nitrogen and phosphorus from wastewater in the circular economy. Bioresour Technol. 2020;300: 122673. https://doi.org/10.1016/j. biortech.2019.122673.

22. Rosemarin A, Macura B, Carolus J, Barquet $K$, Ek F, Järnberg L, Lorick D, Johannesdottir S, Pedersen SM, Koskiaho J, Haddaway NR, Okruszko T. Circular nutrient solutions for agriculture and wastewater-a review of technologies and practices. Curr Opin Environ Sustain. 2020;45:78-91. https://doi.org/10.1016/j.cosust.2020.09.007.

23. Thomas J, Graziosi S, Brunton J, Ghouze Z, O'Driscoll P, Bond M. EPPIReviewer: advanced software for systematic reviews, maps and evidence synthesis. London: EPPI-Centre Software; 2020.

24. van der Wiel BZ, Weijma J, van Middelaar CE, Kleinke M, Buisman CJN, Wichern F. Restoring nutrient circularity: a review of nutrient stock and flow analyses of local agro-food-waste systems. Resour Conserv Recycl X. 2019;3: 100014. https://doi.org/10.1016/j.rcrx.2019.100014.

25. Vaneeckhaute C, Lebuf V, Michels E, Belia E, Vanrolleghem PA, Tack FMG, Meers E. Nutrient recovery from digestate: systematic technology review and product classification. Waste Biomass Valor. 2017;8:21-40. https://doi. org/10.1007/s12649-016-9642-x.

\section{Publisher's Note}

Springer Nature remains neutral with regard to jurisdictional claims in published maps and institutional affiliations. 